\title{
Numerical Evaluation of High-Dimensional Integrals
}

\author{
By T. W. Sag and G. Szekeres
}

1. Introduction. In this paper we describe a practical method for the numerica] evaluation of multiple integrals, designed for high-speed computers. To illustrate the method, consider the integral

$$
\int_{0}^{1} e^{-3 x} d x=0.3167376
$$

We evaluate it by the following three methods:

(a) Simpson's rule

(b) Trapezoid rule

(c) Trapezoid rule, applied to the integral

$$
\begin{aligned}
& \int_{0}^{1} \exp \left\{-\frac{3}{2}(1+\right.\left.\left.\tanh \frac{2 y-1}{4 y(1-y)}\right)\right\} \\
& \cdot\left(\cosh \frac{2 y-1}{4 y(1-y)}\right)^{-2}\left(\frac{1}{2}+y(y-1)\right)(2 y(1-y))^{-2} d y
\end{aligned}
$$

This integral is derived from (1) by the following sequence of transformations:

(i) $x=\frac{1}{2}(1+t)$

(ii) $t=\tanh u /\left(1-u^{2}\right)$

(iii) $u=2 y-1$.

The important step is transformation (ii); the others merely scale down and displace the interval of integration so that the actual interval in terms of the variable $u$ should be $(-1,1)$. Results by the three methods are compared in Table 1 ; the first column gives the number of points used in the calculation.

The results in the last column, although not as good as Simpson, are considerably better than those obtained by the plain trapezoid rule. Reason for the improvement is obvious: the integrand of (2) has the property (shared with the Jacobian of transformation (ii)) that it vanishes, together with all its derivatives, at the endpoints of the interval of integration. As we shall frequently encounter this property, we shall briefly refer to it as property $P$.

For integrands with property $P$ the trapezoid rule is just as good as Simpson. For if $f(x)$ has property $P$ then we can replace $\int_{0}^{1} f(x) d x$ by either of the integrals

$$
\int_{-1 / N}^{1} f^{*}(x) d x, \quad \int_{0}^{1+1 / N} f^{*}(x) d x
$$

where $f^{*}(x)=f(x)$ for $0 \leqq x \leqq 1, f^{*}(x)=0$ for $x<0$ or $x>1$, and $N$ is any positive integer. If Simpson's rule (with $N+1$ subdivisions) is applied to the two

Received July 1, 1963. This work was undertaken at the University of Adelaide by the first author for the degree of M. Sc. and was supported by a Senior Studentship awarded by the Commonwealth Scientific Industrial and Research Organization. 
TABLE 1

\begin{tabular}{c|c|c|c}
\hline & $(\mathrm{a})$ & $(\mathrm{b})$ & (c) \\
\cline { 2 - 3 } 10 & 0.3167517 & 0.3191096 & 0.3185090 \\
20 & 0.3167385 & 0.3173313 & 0.3167146 \\
40 & 0.3167374 & 0.3168858 & 0.3167345 \\
\hline
\end{tabular}

integrals in (3) and the arithmetic means of the two results is taken, we obtain an evaluation of $\int_{0}^{1} f$ by means of the trapezoid rule. That method (a) nevertheless gives better results than (c) is due to the fact that $e^{-3 x}$ is a much milder function than the integrand of (2) which goes down to zero rather violently near the endpoints of the interval.

An explicit expression for the error can be obtained directly from the summation formula of Euler-Maclaurin which, in the case of a function with property $P$, takes the form

$$
\begin{gathered}
\int_{a}^{b} f(x) d x=h \sum_{k=1}^{N} f\left(x_{k}\right)-J_{2 r+1}, \\
x_{k}=a+k h, \quad k=1, \cdots, N, \quad h=\frac{b-a}{N+1}
\end{gathered}
$$

where (for every $r \geqq 0$ )

$$
J_{2 r+1}=\frac{h^{2 r+1}}{(2 r+1) !} \int_{a}^{b} B_{2 r+1}\left(\frac{x-a}{h}\right) f^{(2 r+1)}(x) d x .
$$

$B_{m}(x)$ is the $m$ th Bernoulli polynomial (see [1], p. 526).

The chief merit of method (c) is that it can be extended immediately to higher dimensions. We say that $f(\mathbf{x})$ has property $P_{r}(r>0)$ relative to the ( $n$-dimensional $)$ region $R$ if

$$
f(\mathbf{x})=0, \quad \frac{\partial^{2 k-1}}{\partial x_{i}{ }^{2 k-1}} f(\mathbf{x})=0, \quad k=1, \cdots, r, \quad i=1, \cdots, r
$$

on the boundary of $R$. Then defining $f(\mathbf{x})=0$ outside $R$ we obtain, by repeated application of (4),

$$
\int_{R} f(\mathbf{x}) d \mathbf{x}=h^{n} \sum_{k=1}^{N} f\left(\mathbf{x}_{k}\right)+J_{2 r+1}^{*}
$$

where $\mathbf{x}_{1}, \cdots, \mathbf{x}_{N}$ are equally spaced meshpoints inside $R$, with mesh size $h$, and $J_{2 r+1}^{*}$ is an expression of the form $\frac{1}{(2 r+1) !} h^{2 r+1} K_{2 r+1}$ where $K_{2 r+1}$ is a sum of multiple integrals of functions which are products of Bernoulli polynomials of order $2 r+1$ and partial derivatives of $f(x)$ of order $2 r+1$. If these partial derivatives have a reasonable bound over $R$ and $h$ is small enough, the error term $J_{2 r+1}^{*}$ becomes small and we can apply the simple quadrature formula

$$
\int_{R} f(\mathbf{x}) d \mathbf{x}=h^{n} \sum_{k=1}^{N} f\left(\mathbf{x}_{k}\right)
$$

From the theoretical point of view the existence of an explicit error formula is 
of great importance; it removes the uncertainties usually associated with Monte Carlo type methods. The method is almost as simple as Monte Carlo and generally more accurate. It can be applied to almost any region, though we shall only work out the required transformations for the sphere, cube and simplex. The usefulness of the method is most obvious in higher dimensions (6-20) where hitherto it was practically impossible to evaluate an integral numerically, except by Monte Carlo methods. We have in fact been able to calculate integrals up to 20 dimensions, with reasonable accuracy and tolerable amount of computing time (a few minutes on the IBM 7090). A further advantage of the method is that it can be applied directly to convergent improper integrals in which the integrand becomes infinite on the boundary.

Some typical results will be given in $\$ 3$.

2. Scanning of the Standard Region. To carry out the program described in $\S 1$ it is necessary to find, first, a suitable region $S$ over which the actual integration is performed, and, secondly, a transformation of the given region $R$ into $S$ with the property that the Jacobian of the transformation vanishes in the boundary, together with a convenient number of higher derivatives. All transformations to be discussed in $\S 3$ will in fact have property $P_{\infty}$ relative to $S$.

The selection of the standard region is motivated by two requirements. First, we want $S$ to have a reasonably simple shape so that its points should be given by easily calculable inequalities. Secondly we want a region which can be scanned by a regular lattice which does not contain an intolerably large number of points inside $S$ and yet has a reasonably small mesh size.

The unit cube satisfies the first condition, but not the second one. For example, if in 10 dimensions we take the mesh size as large as $\frac{1}{3}$ of the side length of the cube, the lattice will contain $3^{10}=59049$ meshpoints in the cube. In higher dimensions the situation deteriorates rapidly.

It appears that the only region which fulfils both requirements is the unit sphere

$$
S: x_{i}{ }^{2}+x_{2}^{2}+\cdots+x_{n}^{2} \leqq 1 \text {. }
$$

Scanning of $S$ in lower than 6 dimensions presents no particular problems. In more than 5 dimensions the scanning can be effected as follows:

Take the lattice points of mesh size $h$, situated in the sphere of radius $r_{0}(\leqq 1)$,

$$
x_{1}^{2}+\cdots+x_{n}^{2} \leqq r_{0}^{2}
$$

with coordinates taken from the set of values

$$
\left\{\frac{1}{4} h,-\frac{3}{4} h, \frac{5}{4} h, \cdots,(-1)^{m} \frac{1}{4}(2 m+1) h, \cdots\right\} .
$$

These lattice points fall into a number of successive spherical layers of radii

$$
R_{i}=\frac{1}{4} h(n+8(i-1))^{1 / 2}, \quad i=1, \cdots, N
$$

respectively, where $n$ is the number of dimensions and

$$
N=\left[1+2 r_{0}{ }^{2} / h^{2}-n / 8\right]
$$

is the total number of layers, where $r_{0}$ is the radius of the sphere scanned. For transformations into the unit sphere, which have property $P_{\infty}$, the values of the Jacobian are usually small enough to be neglected outside a radius of 0.8 in the 
TABLE 2

$i$ Number of points

\begin{tabular}{ll}
1 & $\left.\begin{array}{l}1 \\
n \\
1\end{array}\right)$ \\
2 & $\left(\begin{array}{l}n \\
2\end{array}\right)$ \\
3 & $\left(\begin{array}{l}n \\
3\end{array}\right)+\left(\begin{array}{l}n \\
1\end{array}\right)$ \\
& $\left(\begin{array}{l}n \\
4\end{array}\right)+\left(\begin{array}{l}n \\
2\end{array}\right)$ \\
& $\left(\begin{array}{l}n \\
5\end{array}\right)+3\left(\begin{array}{l}n \\
3\end{array}\right)$ \\
5 & $\left(\begin{array}{l}n \\
6\end{array}\right)+4\left(\begin{array}{l}n \\
4\end{array}\right)+\left(\begin{array}{l}n \\
2\end{array}\right)+\left(\begin{array}{l}n \\
1\end{array}\right)$ \\
\hline 6 & $\left(\begin{array}{l}n \\
7\end{array}\right)+5\left(\begin{array}{l}n \\
5\end{array}\right)+3\left(\begin{array}{l}n \\
3\end{array}\right)+2\left(\begin{array}{l}n \\
2\end{array}\right)$ \\
8 & $\left(\begin{array}{l}n \\
8\end{array}\right)+6\left(\begin{array}{l}n \\
6\end{array}\right)+6\left(\begin{array}{l}n \\
4\end{array}\right)+3\left(\begin{array}{l}n \\
3\end{array}\right)$ \\
9 & $\left(\begin{array}{l}n \\
9\end{array}\right)+7\left(\begin{array}{l}n \\
7\end{array}\right)+10\left(\begin{array}{l}n \\
6\end{array}\right)+4\left(\begin{array}{l}n \\
4\end{array}\right)+\left(\begin{array}{l}n \\
3\end{array}\right)+2\left(\begin{array}{l}n \\
2\end{array}\right)$ \\
10 &
\end{tabular}

sphere. Thus when using these transformations, it is only necessary to scan a sphere of radius $r_{0}=0.8$. This permits a considerable saving in computation time (or, for given computation time, a decrease in mesh size by a factor of 0.8 ) as the number of lattice points in the $i$ th layer increases rapidly with $i$ and even the omission of a few layers is a significant saving.

Table 2 gives the number of points in each successive layer.

To obtain these expressions we remark that the coordinates of the points in a given layer are made up of permutations of one or more combinations of the set (9). Thus in layer 3 the only combination which gives radius $R_{3}$ is $\left(-\frac{3}{4} h,-\frac{3}{4} h, \frac{1}{4} h\right.$, $\left.\cdots, \frac{1}{4} h\right)$ and this set has $\left(\begin{array}{l}n \\ 2\end{array}\right)$ distinct permutations. In layer 7 there are four combinations, namely (i) six $-\frac{3}{4} h$-coordinates, (ii) three $-\frac{3}{4} h$-coordinates, one $\frac{5}{4} h$-coordinate, (iii) two $\frac{5}{4} h$-coordinates, (iv) one $-\frac{7}{4} h$-coordinate, all other coordinates $\frac{1}{4} h$. The respective numbers of different permutations of these combinations are $\left(\begin{array}{l}n \\ 6\end{array}\right), 4\left(\begin{array}{l}n \\ 4\end{array}\right),\left(\begin{array}{l}n \\ 2\end{array}\right),\left(\begin{array}{l}n \\ 1\end{array}\right)$.

From Table 2 we find that if for example $n=15$ then 6 layers contain 6534 meshpoints, 7 layers 17119 points, 8 layers 40144 points and 9 layers 147799 points. With $r_{0}=0.8$ and 6 layers the allowable mesh size is $h=3.2 / \sqrt{15+8 \times 5} \simeq 0.4$, 
with 7 layers $h=0.38$. To achieve the same mesh sizes when all points inside $S$ are scanned, 11 and 13 layers would have been necessary, resulting in enormously large numbers of meshpoints.

One drawback of the mesh with coordinates taken from the set $(9)$ is that it is strongly biased relative to the axis pointing in the direction of $(1,1, \cdots, 1)$. This bias can be largely compensated if we use two meshes simultaneously, one with the coordinate set (9) and another one with the set

$$
\left\{-\frac{1}{4} h, \frac{3}{4} h,-\frac{5}{4} h, \cdots(-1)^{m+1} \frac{1}{4}(2 m+1) h, \cdots\right\} .
$$

This is equivalent to integrating the even function $\frac{1}{2}(f(\mathbf{x})+f(-\mathbf{x}))$ over either of these meshes. All results in $\$ 3$ were obtained in this manner.

3. Transformations of the $n$-Sphere, Cube and Simplex. The most common regions over which multiple integrals are evaluated are the sphere, cube and simplex. In this section we shall describe suitable transformations for these regions.

Let $\xi_{1}, \cdots, \xi_{n}$ be cartesian coordinates of the original region $R$, and $x_{1}, \cdots, x_{n}$ the cartesian coordinates of the transformed (normal) region $S$.

(a) $n$-sphere. If $R$ is a sphere with center 0 and radius $\sigma$ we can use a radial transformation

$$
\rho=\sigma g(r), \quad \theta_{i}=\phi_{i} \quad(i=1, \cdots, n-1)
$$

where $g(0)=0, g(1)=1$,

$$
\rho=\left(\xi_{1}{ }^{2}+\cdots+\xi_{n}{ }^{2}\right)^{1 / 2}, \quad r=\left(x_{1}{ }^{2}+\cdots+x_{n}{ }^{2}\right)^{1 / 2}
$$

and $\theta_{i}, \phi_{i}$ are the Euler angles in the $\xi$, y spaces respectively. Such a transformation

TABLE 3

\begin{tabular}{c|c|c|c|c|c}
\hline $\begin{array}{c}\text { Dimen- } \\
\text { sion }\end{array}$ & $\begin{array}{c}\text { Number of } \\
\text { points }\end{array}$ & Computed value & Exact value & \% Error \\
\cline { 2 - 3 } 2 & 177 & 0.785415 & 0.785396 & \\
3 & 528 & 0.523602 & 0.523599 & 0.002 \\
4 & 1272 & 0.308425 & & 0.308424 & 0.000 \\
5 & 1308 & 0.164515 & 0.164493 & 0.000 \\
6 & 1874 & $0.807442 \times 10^{-1}$ & $0.807455 \times 10^{-1}$ & 0.013 \\
7 & 3617 & $0.369114 \times 10^{-1}$ & $0.369122 \times 10^{-1}$ & 0.002 \\
8 & 6196 & $0.158538 \times 10^{-1}$ & $0.158543 \times 10^{-1}$ & 0.002 \\
9 & 9011 & $0.644234 \times 10^{-2}$ & $0.644222 \times 10^{-2}$ & 0.002 \\
10 & 11378 & $0.249079 \times 10^{-2}$ & $0.249039 \times 10^{-2}$ & 0.016 \\
11 & 12145 & $0.919592 \times 10^{-3}$ & $0.919973 \times 10^{-3}$ & 0.041 \\
12 & 20585 & $0.325871 \times 10^{-3}$ & $0.325992 \times 10^{-3}$ & 0.038 \\
13 & 17239 & $0.111153 \times 10^{-3}$ & $0.111161 \times 10^{-3}$ & 0.008 \\
14 & 11873 & $0.365748 \times 10^{-4}$ & $0.365762 \times 10^{-4}$ & 0.004 \\
15 & 17119 & $0.116379 \times 10^{-4}$ & $0.116407 \times 10^{-4}$ & 0.024 \\
16 & 24245 & $0.358487 \times 10^{-5}$ & $0.359086 \times 10^{-5}$ & 0.167 \\
17 & 11731 & $0.107385 \times 10^{-5}$ & $0.107560 \times 10^{-5}$ & 0.163 \\
18 & 15388 & $0.312874 \times 10^{-6}$ & $0.313362 \times 10^{-6}$ & 0.156 \\
19 & 19932 & $0.887768 \times 10^{-7}$ & $0.889236 \times 10^{-7}$ & 0.165 \\
20 & 25520 & $0.245692 \times 10^{-7}$ & $0.246114 \times 10^{-7}$ & 0.172 \\
\hline
\end{tabular}


has the obvious advantage that at all points of the layer (10) the Jacobian has the same value and so it is only necessary to compute it once for each layer.

The Jacobian of transformation (11) is

$$
\partial(\xi) / \partial(\mathbf{x})=(\rho / r)^{n-1} d \rho / d r=\sigma^{n}(g(r) / r)^{n-1} g^{\prime}(r)
$$

and we want $g^{\prime}(r)$ to vanish, together with all derivatives, at $r=1$. A suitable function is

$$
g(r)=\tanh \frac{u r}{1-r^{2}}
$$

where $u$ is a constant. The choice of $u$ depends on the function to be integrated; if the function has large values near the origin and drops down further out, a larger value of $u$ will be used than for functions which have large values near the boundary. For functions with fairly evenly distributed values $u=1.5$ was found to give best results.

Table 3 gives results for the constant test function $f(x)=2^{-n}$ for all dimensions from 2 to 20 .

Similar results were obtained with "mild" functions such as $\sum_{i=1}^{n}\left(\xi_{i}-1\right)^{2}$, $\sum_{i=1}^{n} \cos \xi_{i}, \quad \sum_{i=1}^{n} \exp \xi_{i}$. The Jacobians of the transformations from cube to sphere and simplex to sphere, to be discussed below, supply examples of more irregularly behaving functions; the percentage error grows considerably for these functions and reaches $2-3 \%$ in 15 dimensions with the same number of points used as in Table 3.

(b) n-cube. We assume the $n$-cube in the normal form

$$
-1 \leqq \xi_{i} \leqq 1,
$$$$
i=1, \cdots, n \text {. }
$$

To transform it into the unit $x$-sphere we use the transformation

$$
\xi_{i}=\tanh \frac{u x_{i}}{1-r} \quad(i=1, \cdots, n)
$$

where $r=\left(x_{1}{ }^{2}+\cdots+{x_{n}}^{2}\right)^{1 / 2}$ and $u$ is a suitable parameter. The Jacobian is

$$
\partial(\xi) / \partial(x)=u^{n}(1-r)^{-n-1} \prod_{i=1}^{n}\left(1-\xi_{i}{ }^{2}\right)
$$

TABLE 4

\begin{tabular}{c|c|c|c}
\hline Dimension & Number of points & Computed value & \% Error \\
\cline { 2 - 3 } 6 & 1308 & 1.00032 & 0.032 \\
6 & 1874 & 0.99985 & 0.015 \\
8 & 3617 & 0.99971 & 0.029 \\
9 & 6196 & 0.99956 & 0.044 \\
10 & 9011 & 0.99911 & 0.089 \\
11 & 11378 & 0.99754 & 0.246 \\
12 & 12145 & 0.99548 & 0.452 \\
13 & 10916 & 0.99163 & 0.837 \\
14 & 17239 & 0.98924 & 1.08 \\
15 & 11873 & 0.97937 & 2.06 \\
& 17119 & 0.97461 & 2.54 \\
\hline
\end{tabular}


TABLE 5

\begin{tabular}{c|c|c|c}
\hline Dimension & Computed value & $\frac{1}{2} n_{\pi}$ & \% Error \\
\cline { 2 - 3 } 5 & 7.8541 & 7.8540 & 0.00 \\
6 & 9.3977 & 9.4248 & 0.29 \\
7 & 10.982 & 10.996 & 0.14 \\
8 & 12.539 & 12.566 & 0.22 \\
9 & 14.054 & 14.137 & 0.59 \\
10 & 15.565 & 15.708 & 0.91 \\
11 & 17.104 & 17.279 & 1.01 \\
12 & 18.511 & 18.850 & 1.80 \\
13 & 19.977 & 20.420 & 2.17 \\
14 & 21.071 & 21.991 & 4.18 \\
15 & 22.441 & 23.562 & 4.77 \\
\hline
\end{tabular}

where $\xi_{i}$ is given by (15). Although the factor $(1-r)^{-n-1}$ becomes infinite on the boundary of the sphere, the factors $\left(1-\xi_{i}{ }^{2}\right)$ are of the order $e^{-1 /(1-r)}$ as $r \rightarrow 1$ and the Jacobian and its partial derivatives vanish at $r=1$. Since the transformation is coordinate symmetrical, the Jacobian has to be computed only for a comparatively small number of coordinate combinations taken from the set (9). For ordinary functions $u=1.5$ was again found to be the most suitable value.

Table 4 gives results for $f(z)=1 / 2^{n}$, from 5 to 15 dimensions. The exact value of the integral is of course 1 .

The results in Table 4 are fairly typical for well-behaved functions; a large number of such functions (mainly of the $\sum_{i=1}^{n} \phi\left(\xi_{i}\right)$ type) were tested and the error was found to be of the same order for all functions. The error increased moderately when improper integrals were tested. As a typical example we take $f(\xi)=\sum_{i=1}^{n}\left(1-\xi_{i}^{2}\right)^{-1 / 2}$. The value of the integral is $\frac{1}{2} n \pi$. Results are shown in Table 5 .

(c) $n$-simplex. We had some difficulty in finding suitable transformations from the simplex to the sphere. Assuming the simplex in the form

$$
\xi_{1}+\xi_{2}+\cdots+\xi_{n} \leqq 1, \quad \xi_{i} \geqq 0 \quad(i=1, \cdots, n)
$$

the most obvious idea was to transform (17) into the positive orthant $t_{i} \geqq 0$ $(i=1, \cdots, n)$ by

$$
\xi_{i}=t_{i} /\left(1+\sum_{j=1}^{n} t_{j}\right) \quad(i=1, \cdots, n)
$$

then into the whole $y$-space by

$$
t_{i}=\exp y_{i} \quad(i=1, \cdots, n)
$$

and finally into $S$ by

$$
\rho=\frac{u r}{1-r^{2}}, \quad \rho^{2}=y_{1}^{2}+\cdots+y_{n}^{2}, \quad r^{2}=x_{1}{ }^{2}+\cdots+x_{n}{ }^{2} .
$$

However, the distribution of the resulting Jacobian over the sphere was very uneven and gave bad results, so that the transformation was finally abandoned. 
To obtain a more useful transformation, let us assume the simplex to be in the normal form

$$
0 \leqq \xi_{1} \leqq \xi_{2} \leqq \cdots \leqq \xi_{n} \leqq 1 .
$$

This can be transformed into the cube

$$
0 \leqq y_{i} \leqq 1
$$$$
(i=1, \cdots, n)
$$

by

$$
\xi_{i}=y_{i} y_{i+1} \cdots y_{n}
$$$$
(i=1, \cdots, n)
$$

with the Jacobian

$$
\partial(\xi) / \partial(\mathbf{y})=y_{2} y_{3}^{2} \cdots y_{n}{ }^{n-1},
$$

thus reducing the problem to one of integration over a cube. However (20) is an exceedingly bad function to integrate, especially in higher dimensions; for the maximum of the function is 1 , reached at the top corner of the cube, but its average value is as low as $1 / n$ !

To overcome this difficulty we transform the y-cube into yet another cube

$$
0 \leqq t_{i} \leqq 1
$$$$
(i=1, \cdots, n)
$$

by means of

$$
t_{1}=y_{1}, \quad t_{2}=y_{2}^{2}, \quad \cdots, \quad t_{n}=y_{n}{ }^{n}
$$

for then

$$
\partial(\mathbf{y}) / \partial(\mathbf{t})=\left(n ! y_{2} y_{3}^{2} \cdots y_{n}^{n-1}\right)^{-1}
$$

so that the composite transformation

$$
\xi_{i}=\prod_{j=1}^{n} t_{j}^{1 / j}
$$

has a constant Jacobian,

$$
\partial(\xi) / \partial(\mathbf{t})=1 / n ! .
$$

Finally the $\mathrm{t}$-cube is transformed into the standard cube $-1 \leqq \xi_{i}^{\prime} \leqq 1$, $(i=1, \cdots, n)$ by $\xi_{i}^{\prime}=2 t_{i}-1(i=1, \cdots, n)$, and the $\xi^{\prime}$-cube into $S$ by transformation (15). Since the transformation $\xi \rightarrow \xi^{\prime}$ has constant Jacobian

$$
\partial(\xi) / \partial\left(\xi^{\prime}\right)=1 / 2^{n} n !
$$

the accuracy of integrating the function $f(\xi)=$ const. over the simplex is the same as that given in Table 4. Integrals of various other functions gave very similar accuracies.

4. Conclusion. The method described in this paper can be applied with confidence for the numerical evaluation of multiple integrals over spheres, cubes, simplexes and similar regions up to 20 dimensions. Normally, not more than 20000 points are required to achieve results with an error of at most a few per cent.

Hammer, Stroud and Wymore [2], [3] and more recently Mustard, Lyness and 
Blatt [4] have given integration formulas which could perhaps be extended to higher dimensions. However no actual examples of evaluations of higher dimensional integrals by these methods have been given.

The only method which seems to have been used so far in dimensions higher than 5 is Monte Carlo. The usual version of the method gives bad results, see [5]. An improved version was recently suggested by Haselgrove [6], based on equidistributed sequences. The method gave about 0.003 accuracy when 1000 points were used to compute the 5-dimensional integral

$$
\int_{0}^{1} \cdots \int_{0}^{1} e^{-x_{1} x_{2} \cdots x_{5}} d x
$$

The goodness of this result is somewhat deceptive as the value of the integrand is very nearly unity for most of the region. In fact if the constant function 1 is integrated by this (and practically all available) methods then the exact value is obtained. With our present method a constant test function can be used with just as much reliance as any other well behaved function.

University of Manchester University of New South Wales,

Kensington, New South Wales

1. K. Knopp, Infinite Series, Blackie and Son, Glasgow, 2nd ed., 1944.

2. P. C. Hammer \& A. W. WyMORe, "Numerical evaluation of multiple integrals I," $M T A C$., v. 11, 1957, p. 59-67.

3. P. C. Hammer \& A. H. Stroud, "Numerical evaluation of multiple integrals II," MTAC., v. 12,1958 , p. $272-280$.

4. D. Mustard, J. N. Lyness \& J. M. Blatt, "Numerical quadrature in $n$ dimensions," Comput. J., v. 6, 1963, p. 75-85.

5. P. Davis \& P. Rabinowitz, "Some Monte Carlo experiments in computing multiple integrals," $M T A C$, v. 10,1956 , p. 1-8.

6. C. B. Haselgrove, "A method for numerical integration," Math. Comp., v. 15, 1961, p. $323-337$. 